\title{
TREATMENT OF ENDOTHELIAL DYSFUNCTION WITH ROSUVASTATIN IN PATIENTS WITH PSORIASIS
}

\author{
Olena I. SARIAN ${ }^{1}$, Liudmyla A. BOLOTNA ${ }^{1}$ \\ ${ }^{1}$ Department of Dermatovenereology, Kharkiv Medical Academy of Postgraduate Education, Ukraine \\ Received 18 Oct 2018, Accepted 25 Nov 2018 \\ https://doi.org/10.31688/ABMU.2019.54.1.03
}

\begin{abstract}
Introduction. Psoriasis is an immune-mediated inflammatory skin disease. Since the use of therapeutic agents is often hampered by low efficacy and/or adverse effect profile, the presence of comorbid patholo$\mathrm{gy}$, it is necessary to develop new therapeutic approaches for psoriasis.
\end{abstract}

The objective of the study was to evaluate the clinical and laboratory efficacy of rosuvastatin in the treatment of patients with chronic plaque psoriasis.

Material and methods. The study involved $52 \mathrm{pa}-$ tients with mild and moderate psoriasis, aged 45-64 years. The blood serum level of interleukin-6 (IL-6), high-sensitivity C-reactive protein (hs-CRP) and vascular endothelial growth factor (VEGF) were determined by immunoassay analysis. The serum lipid profile was also determined. Patients were divided into two groups, depending on the method of therapy. Patients of the $1^{\text {st }}$ group received a combination therapy - rosuvastatin, topical corticosteroids (TCS) or phototherapy, patients of the $2^{\text {nd }}$ group were prescribed only TCS or phototherapy.

Results. The clinical improvement of psoriasis is noted under the influence of combination therapy. The PASI (Psoriatic Area and Severity Index) score decreased in 3 months by $68.2 \%$. A marked decrease in the level of IL-6, hs-CRP after 1 month was noted, less

\section{RésumÉ}

Traitement des dysfonctionnements endothéliaux chez les patients atteints de psoriasis par la rosuvastatine

Introduction. Le psoriasis est une maladie inflammatoire de la peau à médiation immunitaire. Dans la mesure où l'utilisation des agents thérapeutiques s'avère souvent inefficace et/ou peut provoquer des effets indésirables et s'accompagner d'une pathologie comorbide, il est nécessaire de développer de nouvelles approches thérapeutiques dans le traitement du psoriasis. L'objectif de cette étude consistait à évaluer l'efficacité clinique de la rosuvastatine et celle prouvée en laboratoire dans le traitement des patients atteints de psoriasis chronique en plaques.

Matériel et méthodes. 52 patients âgés de 45 à 64 ans atteints de psoriasis léger ou modéré ont participé à cette étude. Les taux sériques d'interleukine-6 (IL-6), de protéine C-réactive à haute sensibilité (hsC-RP) et de facteur de croissance endothéliale vasculaire (VEGF) ont été déterminés par un dosage immunologique. Les paramètres de la concentration des lipides sériques sont déterminés par des analyses biochimiques. En fonction de la méthode de traitement, les patients ont été divisés en deux groupes. Les patients du premier groupe ont reçu un traitement qui combinait de la 
significant VEGF relative to the results before therapy. Lipid metabolism indices changed reliably only at the end of the $3^{\text {rd }}$ month - the levels of TC and LDL-C were reduced.

Conclusions. The use of rosuvastatin in the combination therapy of patients with psoriasis increases the effectiveness of treatment, helps to reduce the level of inflammatory markers, proangiogenic growth factor and normalize blood lipid spectrum.

Keywords: psoriasis, endothelial dysfunction markers, treatment, statins.

Abbreviations: BMI - Body Mass Index; SAP systolic arterial pressure; CVDs - cardiovascular diseases; DAP - diastolic arterial pressure; HDL-C high-density lipoprotein cholesterol;HMG-CoA Hydroxy-3-methylglutaryl coenzyme A reductase; hsC-RP -high-sensitivity C-reactive protein; IL - interleukin; LDL-C -low-density lipoprotein cholesterol; PASI - Psoriatic Area and Severity Index; SCORE Systematic COronary Risk Evaluation; TC - total cholesterol; TCS - topical corticosteroid; TG - triglyceride; VEGF - vascular endothelial growth factor.

\section{INTRODUCTION}

Endothelial dysfunction is one of the universal mechanisms of the pathogenesis of many diseases (atherosclerosis, arterial hypertension, diabetes mellitus, etc. $)^{1}$. Currently, it is obvious that the vascular endothelium is an active metabolic system that supports vascular homeostasis through a number of important functions: modulating the vascular tone, forming the extracellular matrix, regulating chemotactic, inflammatory, and reparative processes in response to local damage, etc. The predominant violation of one or another endothelium function depends on the localization of pathological processes, the predominance of certain inflammatory mediators, the presence of hemodynamic changes. It is important to determine the mediators of endothelial damage in the blood, whose level correlates with endothelial dysfunction (C-reactive protein, homocysteine, reactive oxygen species and others).

Psoriasis is a chronic inflammatory skin disorder, immunologically characterized by the presence of IL-23 /Th17 axis $^{2-3}$. Patients with psoriasis are at higher risk of developing „systemic“ associated diseases, such as cardiovascular diseases (CVDs), metabolic syndrome, and obesity $y^{4.5}$. The reason for the association of psoriasis with other diseases remains the subject of scientific debate, however, in these pathological conditions, activation of generalized nonspecific rosuvastatine et des corticostéroïdes topiques (TCS) ou des séances de photothérapie, les patients du deuxième groupe se sont vus prescrire uniquement des corticostéroïdes topiques (TCS) ou des séances de photothérapie. Résultats. Une amélioration clinique du psoriasis a été constatée dans les cas du traitement combiné. Sur une période de 3 mois, le score PASI (indice de sévérité du psoriasis) a diminué de $68,2 \%$. Au bout d'un mois, on a noté une baisse importante du taux d'IL-6 et de hsC-RP, ainsi qu'une diminution de VEGF, par rapport aux résultats obtenus avant le traitement. Les indices du métabolisme lipidique n'ont changé de façon stable qu'à la fin du troisième mois: les taux de TC et de LDL-C ont été réduits.

Conclusions. L'utilisation de la rosuvastatine dans le cadre du traitement combiné chez les patients atteints de psoriasis augmente l'efficacité du traitement, aide à réduire le niveau des marqueurs inflammatoires, le facteur de croissance pro angiogénique et à normaliser le spectre lipidique sanguin.

Mots-clés: psoriasis, marqueurs de dysfonctionnement endothélial, traitement, statines.

inflammation and endothelial dysfunction are likely to occur.

There are few data about the functional state of the vascular endothelium in psoriasis. Endothelial dysfunction in patients with psoriasis and arterial hypertension may be due to impaired oxidative metabolism of L-arginine and is manifested by a decrease in the bioavailability of $\mathrm{NO}$ and a high level of inactivation, oxidative stress and impaired antioxidant status $^{6}$. The increased activity of von Willebrand factor, endothelin-1 has been established in male patients, especially with the common process and combination with metabolic syndrome $e^{7}$. The function of the endothelium is impaired in patients with psoriasis, the intima-media layer is thickened compared with healthy individuals, which makes psoriasis an independent factor of subclinical atherosclerosis ${ }^{8}$.

Typically, dyslipidemia presents as increased low-density lipoprotein (LDL), VLDL, and triglyceride (TG) levels and decreased high-density lipoprotein (HDL) levels. Several mechanisms underlying the association between dyslipidemia and psoriasis are the activation of Th1 cells, autoantibodies recognizing oxidized LDL, and psoriasis medications such as oral retinoids and cyclosporine. Specifically, the cytokines interleukin (IL)-1, IL-6, and tumor necrosis factor alpha that mediate psoriasis may alter the function of hepatocytes and arterial smooth muscle cells, resulting in altered lipoprotein compositions, 
enhanced expression of cellular adhesion molecules, and increased lipid deposition on arterial walls.

The mainstay of psoriasis treatment involves topical agents (e.g., corticosteroids and vitamin D analogs) for milder disease and systemic therapy (e.g., oral immunosuppressants, phototherapy, or biological agents) for more severe disease ${ }^{9}$. Because the use of therapeutics is often hampered by low efficacy and/or adverse effect profile, the presence of comorbid pathology, a need to develop novel therapeutic approaches for psoriasis is increasing.

3-Hydroxy-3-methylglutaryl coenzyme A (HMG-CoA) reductase inhibitors, more commonly referred to as statins, a class of cholesterol-lowering agents, are used for the treatment of dyslipidaemia and reduction of atherosclerotic CVD risk. Their broad and potent effects on the lipid profile, in conjunction with cholesterol-independent (pleiotropic) cardioprotective effects, have resulted in statins being amongst the most highly prescribed medications worldwide. Recent research has proven that statins also have immunomodulatory and anti-inflammatory activities and thus they can be used in the treatment of several immunological conditions which are characterized by a Th1 immune response ${ }^{10}$. There are few publications about the efficacy and safety of simvasta$\operatorname{tin}^{11-13}$ and atorvastatin ${ }^{14-15}$ in patients with psoriasis. Clinical improvement results from combination therapy, including narrow-band UV therapy, rosuvastatin and omega-3 polyunsaturated fatty acids for the treatment of moderate psoriasis associated with metabolic syndrome ${ }^{16}$.

The obJective OF THE STUdY was to evaluate the clinical and laboratory efficacy of rosuvastatin in the treatment of patients with chronic plaque psoriasis.

\section{Material AND Methods}

The study included 52 patients with chronic plaque psoriasis, 28 men and 24 women, aged 45-64 years. All patients signed an informed consent to participate in the study.

The prospective study took place in the Dermatovenereologic Dispensary No. 4 of Kharkiv (clinical base of Kharkiv Medical Academy of Postgraduate Education), Ukraine, between January 2015 and December 2017.

The criteria for inclusion in the study were the following: the presence of clinical manifestations of chronic plague psoriasis, age over 45 years, the level of total blood cholesterol more than $5.2 \mathrm{mmol} / \mathrm{L}$, the absence of chronic somatic diseases or their exacerbations at the time of examination of patients. Exclusion criteria were the following: the presence of inflammatory and endocrine diseases, surgical interventions in the last 3 months, hypertriglyceridemia $>4.5 \mathrm{mmol} / \mathrm{L}$, using lipid-lowering drugs less than 1 month before being included in the study, increasing the level of liver transaminases or creatine kinase more than twice the normal values.

PASI (Psoriatic Area and Severity Index) score was used to measure psoriasis severity, SCORE (Systematic Coronary Risk Evaluation) - to measure total coronary risk, BMI (Body Mass Index) -to assess the degree of compliance of a person's mass and height.

The level of serum IL-6 (pg/mL), vascular endothelial growth factor (VEGF, pg/mL), high-sensitivity C-reactive protein (hs-CRP, $\mathrm{mg} / \mathrm{L}$ ) were determined by ELISA-BEST (Vector-Best, Novosibirsk, Russia).The serum lipids have been also determined: total cholesterol (TC), TG, HDL-cholesterol (HDL-C), LDL-cholesterol (LDL-C) and aspartate aminotransferase, alanine aminotransferase, creatine kinase, total bilirubin.

Patients were divided into two groups depending on the method of therapy. Patients of group 1 ( $\mathrm{n}=$ 30 ) received a combination therapy - rosuvastatin 10 mg daily for 12 weeks, topical corticosteroids (TCS) (mometasone furoate cream) with PASI $>10$ or phototherapy with PASI $>10$. Patients of group $2(n=22)$ were prescribed only topical therapy or phototherapy. The phototherapy (narrowband UVB-therapy, 311 $\mathrm{mm}$ emission) using Psorolight 100-6cabin (Poltava, Ukraine).Clinical and laboratory examinations were performed at the onset of treatment, 1 and 3 months after the treatment onset.

The control group consisted of 30 healthy individuals(volunteers) of the same age and sex.

Statistical analyses in this study were conducted using MS Excel and STATISTICA 8.0 software. The mean (M) and standard error (m) for each indicator were used to determine the difference between the values of the indicators in the comparison groups, using Student's t-criteria. The difference between groups was considered statistically significant when $\mathrm{p}<0.05$. The correlation between the numerical indices was estimated by the Spearman correlation coefficients.

\section{Results}

Plaque psoriasis in all patients had a chronic relapsing course. Mild psoriasis was diagnosed in 19 (36.5\%) patients, moderate psoriasis - in 33 (63.5\%) patients. Maximum and minimum PASI scores were 5.8 and 14.7 , averaged $12.1 \pm 1.2$. The average duration of the disease was $6.7 \pm 0.8$ years, patients with a recurrence rate $1-2$ times a year prevailed. The lesion of the nail plates was detected in 24 (46.1\%) patients. CVDs 
Treatment of endothelial dysfunction with rosuvastatin in patients with psoriasis - SARIAN et al

Table 1.Indicators of endothelial dysfunction in patients with psoriasis before treatment $(\mathrm{M} \pm \mathrm{m})$

\begin{tabular}{ccc}
\hline Indicator, unit & Patients, $n=52$ & Control group, $n=30$ \\
\hline IL-6, $\mathrm{pg} / \mathrm{ml}$ & $5.96 \pm 0.81^{* *}$ & $1.07 \pm 0.10$ \\
\hline hsC-RP, $\mathrm{mg} / \mathrm{l}$ & $6.40 \pm 0.67^{* *}$ & $0.70 \pm 0.06$ \\
\hline VEGF, $\mathrm{pg} / \mathrm{ml}$ & $382.3 \pm 37.7^{* *}$ & $76.7 \pm 6.2$ \\
\hline TC, $\mathrm{mmol} / \mathrm{l}$ & $7.03 \pm 0.45^{*}$ & $4.48 \pm 0.26$ \\
\hline HDL-C, $\mathrm{mmol} / \mathrm{l}$ & $1.17 \pm 0.05$ & $1.33 \pm 0.06$ \\
\hline LDL-C, $\mathrm{mmol} / \mathrm{l}$ & $4.40 \pm 0.21^{* *}$ & $2.35 \pm 0.19$ \\
\hline TG, $\mathrm{mmol} / \mathrm{l}$ & $3.13 \pm 0.20^{*}$ & $1.87 \pm 0.10$ \\
\hline
\end{tabular}

Note: the differences are significant between the indicators in patients and control group at $\mathrm{p}<0.05-{ }^{*}, \mathrm{p}<0.01-{ }^{* *}$.

(arterial hypertension, coronary heart disease) were established in 20 (38.5\%) patients. Smokers were 32 $(61.5 \%)$ patients with psoriasis and 7 (23.3\%) control subjects. Complaints of pain in the joints or their deformation were not detected in patients with psoriasis.

Systolic arterial pressure (SAP) in patients with psoriasis ranged from 110 to $145 \mathrm{mmHg}$, on average $128.1 \pm 3.5 \mathrm{mmHg}$. SAP in the control group was $122.1 \pm 3.1 \mathrm{mmHg}$. Diastolic arterial pressure (DAP) ranged from 75 to $90 \mathrm{mmHg}$, on average $79.6 \pm 1.5$ $\mathrm{mmHg}$. DAP in practically healthy individuals was $80.1 \pm 2.1 \mathrm{mmHg}$. The CVD risk assessment on the SCORE scale was performed in patients with psoriasis without CVD. The value of SCORE varied from 0.17 to $7.88 \%$, low risk was determined in 14 (34.1\%) patients, medium in $24(58.6 \%)$ and high in $3(7.3 \%)$ patients. Patients with a very high risk of developing CVD were not identified.

BMI in patients was $26.43 \pm 0.7 \mathrm{~kg} / \mathrm{m}^{2}$, in healthy individuals $24.2 \pm 1.1 \mathrm{~kg} / \mathrm{m}^{2}$ ( $\left.\mathrm{p}>0.05\right)$.

The study of inflammatory biomarkers, proangiogenic growth factor in patients with psoriasis revealed a significant increase serum level of IL-6 and hsC-RP, VEGF in patients relative to the values in the control group $(p<0.01)$. The level of most of the indicators of the lipid spectrum was significantly increased in patients, except for HDL-C ( $p>0.05)$ (Table 1). These results suggest endothelial dysfunction in patients with psoriasis.

Psoriasis of moderate severity was accompanied by an increase in PASI score $(14.5 \pm 1.4)$ and level of inflammatory mediators, especially hs-CRP $(9.76 \pm 0.89 \mathrm{mg} / \mathrm{L})$, as well as VEGF $(351.17 \pm 34.28 \mathrm{pg} /$ $\mathrm{mL}$ ) compared with indicators in patients with mild psoriasis (respectively $7.5 \pm 0.6,3.42 \pm 0.41 \mathrm{mg} / \mathrm{L}$ and $191.10 \pm 24.31 \mathrm{pg} / \mathrm{mL}, \mathrm{p}<0.05$ ).

Analysis of parameters depending on comorbidities revealed more significant deviations of IL-6 $(8.57 \pm 0.74 \mathrm{pg} / \mathrm{mL}, \mathrm{p}<0.05)$, hs-CRP $(7.55 \pm 1.23$ $\mathrm{mg} / \mathrm{L}, \mathrm{p}<0.05)$, the tendency to an increase in the level VEGF and TG ( $p>0.05)$ in patients with CVDs compared with those in patients without CVDs (IL-6 $4.79 \pm 0.33 \mathrm{pg} / \mathrm{mL}$, hs-CRP $4.41 \pm 0.46 \mathrm{mg} / \mathrm{L})$. The level of serum lipids in patients of different groups

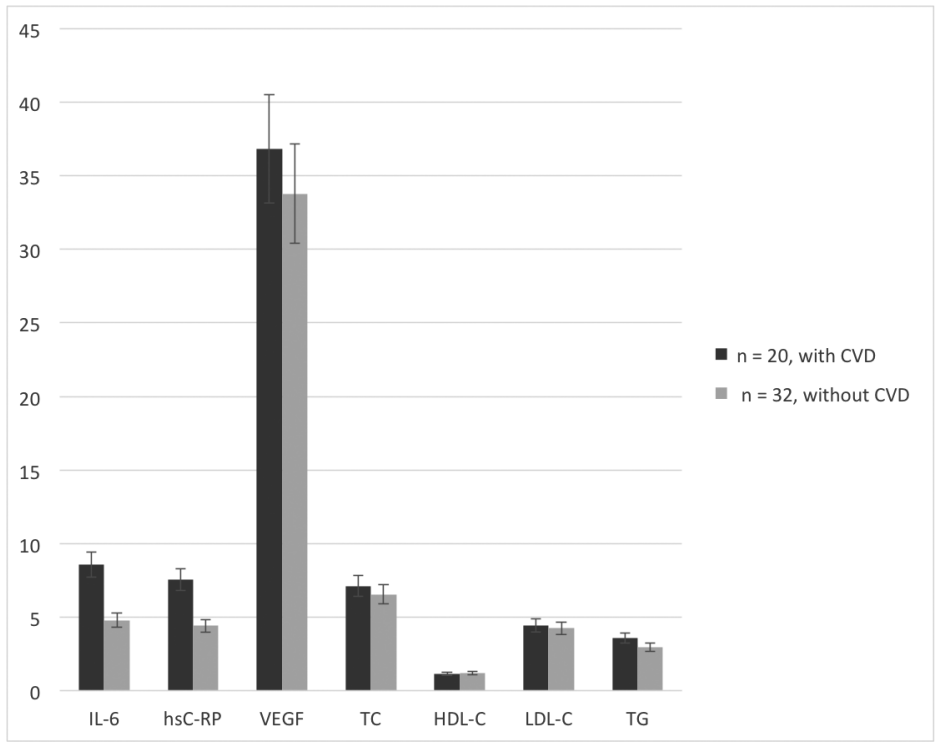

Figure 1 


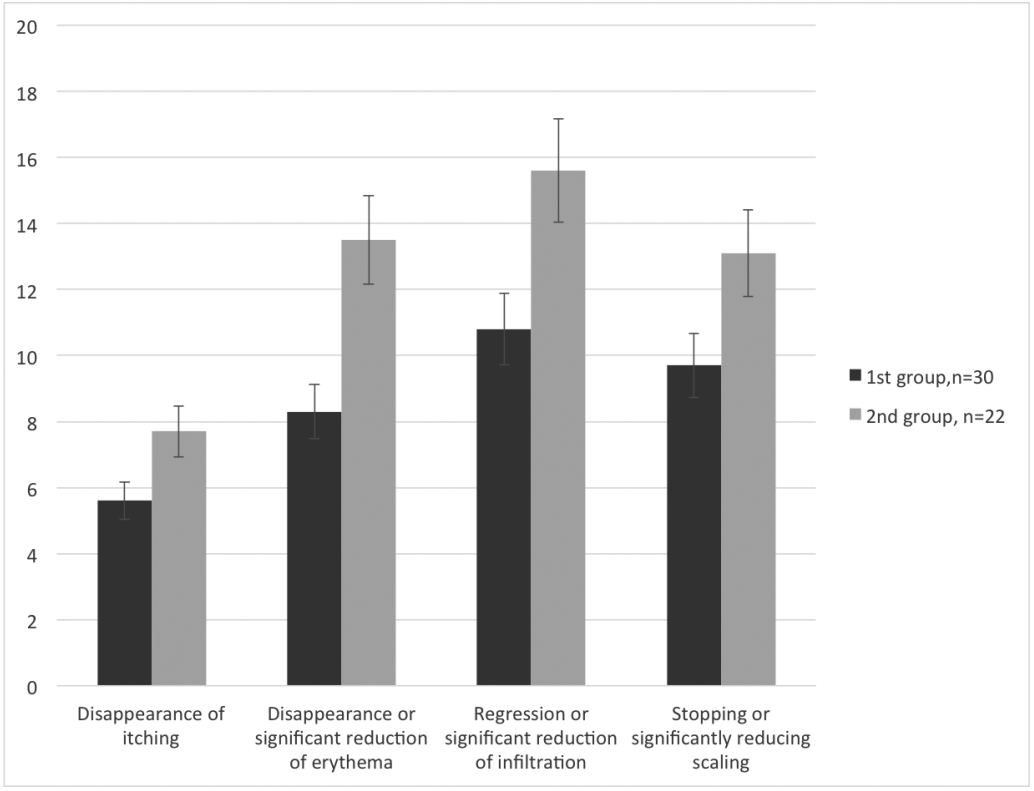

Figure 2

did not significantly change during the $1^{\text {st }}$ month of therapy (Figure 1).

The analysis of indicators after treatment was further carried out in patients without CVDs, so in both groups the number and nature of the pathology was almost the same. In the $1^{\text {st }}$ group, mild psoriasis was detected in $11(36.7 \%)$ patients, moderate- in $19(63.3 \%)$ patients, in the $2^{\text {nd }}$ group - in $8(36.4 \%)$ and $14(63.6 \%)$ patients respectively. PASI score in patients of the $1^{\text {st }}$ group was $12.9 \pm 1.1$, PASI score in patients of the $2^{\text {nd }}$ group $12.1 \pm 0.9$. CVDs were established in $12(40.0 \%)$ patients of the $1^{\text {st }}$ group, 8 $(36.6 \%)$ patients of the $2^{\text {nd }}$ group.

The use of rosuvastatin in the complex treatment of patients with psoriasis contributed to the clinical improvement of psoriasis and the normalization of most of laboratory parameters. The evolution of the main dermatological symptoms of dermatosis occurred at different times under the influence of therapy (Figure 2), draws attention to a shorter period of regression of erythema and infiltration in patients of the $1^{\text {st }}$ group $(8.3 \pm 0.6$ and $10.8 \pm 0.7$ days, $p<0,05)$ compared to those of patients in the $2^{\text {nd }}$ group (respectively $13.5 \pm 0.9$ and $15.6 \pm 1.2$ days). Itching and scaling of the skin disappeared in patients of the 1st group $(5.6 \pm 0.4$ and $9.7 \pm 0.9$ days) and $2^{\text {nd }}$ group ( $7.7 \pm 0.6$ and $13.1 \pm 0.8$ days) at approximately the same time ( $>0.05)$.

An analysis of the reduction of PASI score in patients of different therapeutic groups in 1 month after therapy also allowed identifying the advantage of a complex treatment method using a lipid-lowering drug rather than monotherapy with TCS or phototherapy. PASI score in the $1^{\text {stgroup was }} 7.9 \pm 0.6$, in the $2^{\text {nd }}$ group $10.8 \pm 0.9(\mathrm{p}<0.05)$

Laboratory indices have undergone positive changes in patients of the $1^{\text {st }}$ and $2^{\text {nd }}$ groups after treatment. Serum level of immune-inflammatory parameters, IL-6 $(2.34 \pm 0.35 \mathrm{pg} / \mathrm{mL})$ and hsC-RP $(2.11 \pm 0.30 \mathrm{mg} / \mathrm{L})$, as well as VEGF $(227.1 \pm 10.1 \mathrm{pg} /$ $\mathrm{mL}$ ), decreased 1 month after treatment ( $\mathrm{p}<0.05$ ). Using only TCS or phototherapy allowed lowering the values of IL-6 $(4.89 \pm 0.48 \mathrm{pg} / \mathrm{mL})$, hs-CRP $(5.70 \pm 0.55 \mathrm{mg} / \mathrm{L}), \operatorname{VEGF}(291.3 \pm 27.2 \mathrm{pg} / \mathrm{mL})$, but their level did not differ from the indicators before treatment ( $p>0.05)$. Significant changes in the parameters of the lipid profile of the blood are not defined in all patients.

After 3 months of therapy (Table 2), all laboratory parameters in patients of the $1^{\text {st }}$ group, except for hs-CRP, VEGF, significantly decreased and did not differ from those in the control group ( $p>0.05)$. Despite the positive dynamics of the indicators in patients of the $2^{\text {nd }}$ group, the levels of IL-6, hs-CRP and VEGF remained elevated relative to the parameters in the control group $(p<0.05)$. Values of lipid profile did not practically change relative to those before treatment $(\mathrm{p}>0.05)$

Normalization or positive dynamics of laboratory parameters occurred simultaneously with the disappearance or decrease in the intensity of manifestations of dermatosis. PASI score in the $1^{\text {st }}$ group of patients was 4.1 \pm 0.5 , in the $2^{\text {nd }}$ group $8.0 \pm 0.8(p<0,05)$.

SAP in patients with psoriasis of the $1^{\text {st }}$ group was $123.1 \pm 3.3 \mathrm{mmHg}$, in the $2^{\text {nd }}$ group was $127.5 \pm 3.5$ $\mathrm{mmHg}$, DAP was respectively $80.1 \pm 1.5 \mathrm{mmHg}$ and 
Table 2. Indicators of endothelial dysfunction in patients with psoriasis in 3 months after the start of treatment $(\mathrm{M} \pm \mathrm{m})$

\begin{tabular}{|c|c|c|c|}
\hline \multirow{3}{*}{ Indicator } & \multicolumn{3}{|c|}{ Patients, $n=52$} \\
\hline & \multicolumn{2}{|c|}{ After treatment } & \multirow{2}{*}{$\begin{array}{c}\text { before } \\
\text { treatment, } n=52\end{array}$} \\
\hline & $1^{\text {st }}$ group, $n=30$ & $2^{\text {nd }}$ group, $n=22$ & \\
\hline $\mathrm{IL}-6, \mathrm{pg} / \mathrm{ml}$ & $1.49 \pm 0.11^{* *}$ & $3.56 \pm 0.42^{*_{\mathrm{x}}}$ & $5.96 \pm 0.81$ \\
\hline hsC-RP, mg/l & $1.78 \pm 0.11^{* *_{\mathrm{x}}}$ & $3.69 \pm 0.35^{* *_{\mathrm{x}}}$ & $6.40 \pm 0.67$ \\
\hline VEGF, pg/ml & $116.2 \pm 6.6^{* * \mathrm{x}}$ & $204.4 \pm 15.1^{* \mathrm{x}}$ & $382.3 \pm 37.7$ \\
\hline $\mathrm{TC}, \mathrm{mmol} / \mathrm{l}$ & $4.64 \pm 0.36^{*}$ & $6.37 \pm 0.41^{x}$ & $7.03 \pm 0.45$ \\
\hline HDL-C, mmol/1 & $1.25 \pm 0.07$ & $1.20 \pm 0.07$ & $1.17 \pm 0.05$ \\
\hline LDL-C, mmol/1 & $2.52 \pm 0.19^{*}$ & $3.68 \pm 0.19^{x}$ & $4.42 \pm 0.21$ \\
\hline $\mathrm{TG}, \mathrm{mmol} / \mathrm{l}$ & $2.39 \pm 0.20$ & $3.33 \pm 0.26^{x}$ & $3.13 \pm 0.20$ \\
\hline
\end{tabular}

Note: differences between the indicators in patients before and after treatment at $\mathrm{p}<0.05-{ }^{*}$,between the indicators in groups of patients and control group at $\mathrm{p}<0.05-\mathrm{x}$.

$76.6 \pm 3.4 \mathrm{mmHg}$. Low and medium risk for SCORE was determined in $11(73.7 \%)$ and 7 (38.9\%) patients of the $1^{\text {st }}$ group, whereas low, medium and high risk was determined respectively in $4(28.6 \%), 9$ (64.3\%) and $1(7.1 \%)$ patient of the $2^{\text {nd }}$ group after therapy. The BMI of patients in the process of therapy has not changed.

The patients tolerated the therapy well; the level of biochemical parameters (transaminases, creatine-kinase and total bilirubin) did not change.

\section{Discussion}

Our experience in the use of rosuvastatin (at a dose of $10 \mathrm{mg}$ ) in the complex treatment of patients with plaque psoriasis at the age of $45 \mathrm{y}$ indicates not only hypolipidemic, but also anti-inflammatory and antiangiogenic effects of the drug. Rosuvastatin is a statin of the last generation, a fully synthetic inhibitor of HMG-CoA reductase ${ }^{17-18}$. The drug has the longest half-life among all statins and is the only statin that is minimally metabolized by the cytochrome P450 system, and therefore the probability of its interaction with many drugs is low. This property of rosuvastatin facilitates its introduction in the complex therapy of patients. Rosuvastatin molecules are more hydrophilic than most other statins, highly selective for hepatocyte membranes and have a more pronounced inhibitory effect on the synthesis of LDL-C than other statins. One of the main features of rosuvastatin is lipid-lowering efficacy in the initial dose (10 mg per day), which increases with increasing dose. It has been established that the drug is capable of significantly increasing the level of HDL-C (as an independent cardiovascular risk marker) and exceeds the efficiency of atorvastatin. The powerful anti-inflammatory potential of rosuvastatin can be explained by its ability to enter the systemic circulation in very high concentrations, while other statins „work“ only in the liver. Statins lower CRP and tumor necrosis factor alpha levels while downregulating adhesion molecules on leukocytes and endothelial cells and inhibiting major histocompatibility complex II expression and chemokine receptors on Th1 cells.

The clinical improvement of psoriasis is noted under the influence of complex therapy with the inclusion of rosuvastatin. The PASI score decrease in 3 months by $68.2 \%$ and the patient's complaints absence were considered as high efficiency of treatment. The PASI score decreased only by $27.3 \%$ after treatment in patients of the $2^{\text {nd }}$ group. After treatment, a moderate risk of coronary disease decreased by $19.7 \%$ and the absence of a high risk in patients of $1^{\text {st }}$ group, whereas in patients of $2^{\text {nd }}$ group moderate risk increased by $5.7 \%$, the high risk remained at the same level (7.1\%).

Anti-inflammatory properties of rosuvastatin have been revealed after the $1^{\text {st }}$ month of therapy, antiangiogenic and hypolipidemic properties after the $3^{\text {rd }}$ month of treatment. A marked decrease in the level of immune inflammatory biomarkers (IL-6, hs-CRP) after 1 month was noted (by $60.7 \%$ and $67.7 \%$ respectively), less significant VEGF (by 40.6\%) relative to the results before therapy. These parameters continued to decline towards the end of therapy relative to those after the 1st month of treatment: IL- 6 by $36.3 \%$, hs-CRP by $15.6 \%$, VEGF by $48.8 \%$. Lipid metabolism indices changed reliably only at the end of the $3^{\text {rd }}$ month - the level of TC levels and LDL-C was reduced (respectively by $33.6 \%$ and $43 \%$, $\mathrm{p}<0.05)$.

The lack of correlation between hsC-RP, TC and VEGF suggests that a decrease in VEGF is direct, and not mediated through the effect on blood lipids and inflammatory factors, by the effect of the drug. Probably, not only hypolipidemic, but also pleiotropic 
effects (decrease in the activity of inflammation, normalization of angiogenesis) stabilize the psoriatic process, which is confirmed by clinical results. It should be noted that rosuvastatin is well tolerated by patients, side effects, changes in the level of hepatic aminotransferases, total bilirubin were not detected.

\section{Conclusions}

Use of rosuvastatin in the complex therapy of patients with mild and moderate plaque psoriasis increases the effectiveness of therapy, reduces the level of inflammatory markers (IL-6, hs-CRP) after 1 month of therapy, the level of proangiogenic growth factor (VEGF) and normalizes blood lipid spectrum after 3 months of therapy. Statins have a wide spectrum of action and can be an addition to the treatment of psoriasis as an immune-mediated dermatosis, reduce the risk of lipid disorders and CVDs.

\section{Compliance with Ethics Requirements:}

"The authors declare no conflict of interest regarding this article"

„The authors declare that all the procedures and experiments of this study respect the ethical standards in the Helsinki Declaration of 1975, as revised in 2008(5), as well as the national law. Informed consent was obtained from all the patients included in the study"

"No funding for this study"

\section{References}

1. Rajendran P, Rengarajan T, Thangavel J, et al. The vascular endothelium and human diseases. International Journal of Biology 2013; 9(10):1057-1069.

2. Hawkes JE, Chan TC, Krueger JG. Psoriasis pathogenesis and the development ofnovel targeted immune therapies. Journal of Allergy and Clinical Immunology 2017;140(3):645-653.

3. Chiricozzi A, Romanelli P, Volpe E, Borselino G, Romanelli M. Scanning the immunopathogenesis of psoriasis. International Journal of Molecular Sciences 2018;19:179.

4. Davidovici BB, Sattar N, Jorg PC, et. al. Psoriasis and systemic inflammatory diseases. Journal of Investigative Dermatology 2010;130:1785-1796.
5. Ni C, Chiu MW. Psoriasis and comorbidities: links and risks. Clinical, Cosmetic and Investigational Dermatology 2014; 7:119-132.

6. Belovol AN. Vascular endothelial dysfunction in patients with psoriasis (Published in Ukrainian). Ukrainian Journal of Dermatology, Venereolology, Cosmetology 2010;1(36): 17-21.

7. Kaud D. Features of endotoxin- and lipoflavone-dependent lymphoid regulation of the synthesis of cytokines IL-1 $\beta$, IL-4 and TNF $\alpha$ by vascular endothelial cells in patients with psoriasis, occurring in combination with the metabolic syndrome (Published in Ukrainian). Dermatology and venereology 2008;1: 34-39.

8. Bańska-Kisiel K, Haberka M, Bergler-Czop B, et al. Carotid intima-media thickness in patients with mild or moderate psoriasis. Advances in Dermatology and Allergology 2016; XXXIII (4): 286-289.

9. Samarasekera E, Sawyer L, Parnham J, Smith CH. Guideline Development Group. Assessment and management of psoriasis: Summary of NICE guidance. British Medical Journal 2012; 345: e6712.

10. Rajpara AN, Goldner R, Gaspari A. Psoriasis: can statins play a dual role? Dermatology Online Journal 2010;16(2):2.

11. Shirinsky IV, Shirinsky VS. Efficacy of simvastatin in plaque psoriasis: A pilot study. Journal of the American Academy of Dermatology 2007;57: 529-31.

12. Aslam S, Khurshid K, Asad F, et al. Efficacy and safety of simvastatin in chronic plaque psoriasis. Journal of Pakistan Association of Dermatologists 2013; 23: 310-314.

13. Naseri M, Hadipour A, Sepaskhah M, Namazi MR. The remarkable beneficial effect of adding oral simvastatin to topical betamethasone for treatment of psoriasis: A double-blind, randomized, placebo-controlled study. Nigerian Journal of Medicine 2010; 19: 58-61.

14. Faghihi T, Radfar M, Mehrabian Z, Ehsani AH, Hemami RM. Atorvastatin for the treatment of plaque-type psoriasis. Pharmacotherapy 2011;31:1045-1050.

15. Vasiluk IU, Perlamutrov UI, Shkol'nik MN, Shkol'nik EL. Possibilities of atorvastatin in complex management of extensive psoriasis in patients with arterial hypertension (Published in Russian). Kardiologiia 2010; 50: 37-46.

16. Virstyuk N, Nykyforuk M. Clinical efficacy of omega-3 polyunsaturated fatty acids and rosuvastatin in combination therapy for patients with psoriasis and metabolic syndrome. Journal of Pharmaceutical Innovation 2016; 5 (4): 90-92.

17. Semenova AE, Sergienko IV, Kukharchuk VV. Possible mechanisms for the stabilization of atherosclerotic plaque during therapy with rosuvastatin (Published in Russian). Atherosclerosis and dyslipidemia 2010; 1: 20-24.

18. Luvai A, Mbagaya W, Hall AS, Barth JH. Rosuvastatin: a review of the pharmacology and clinical effectiveness in cardiovascular disease. Clinical Medicine Insights Cardiology 2012; 6:17-33. 